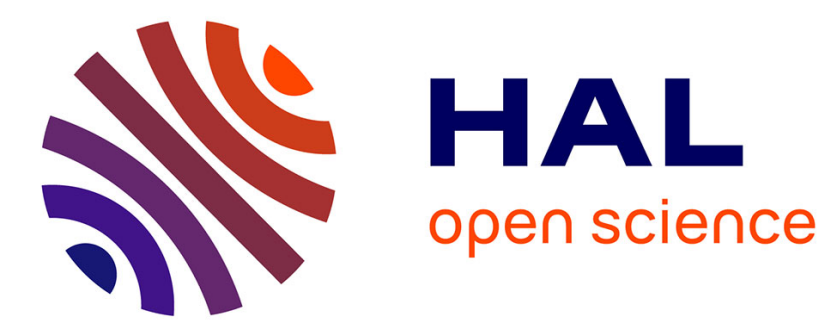

\title{
Probabilistic uncertainty modeling for thermomechanical analysis of plasterboard submitted to fire load
}

S. Sakji, Christian Soize, J.-V. Heck

\section{To cite this version:}

S. Sakji, Christian Soize, J.-V. Heck. Probabilistic uncertainty modeling for thermomechanical analysis of plasterboard submitted to fire load. Journal of Structural Engineering-ASCE, 2008, 134 (10), pp.1611-1618. 10.1061/(ASCE)0733-9445(2008)134:10(1611) . hal-00685092

\section{HAL Id: hal-00685092 \\ https://hal.science/hal-00685092}

Submitted on 4 Apr 2012

HAL is a multi-disciplinary open access archive for the deposit and dissemination of scientific research documents, whether they are published or not. The documents may come from teaching and research institutions in France or abroad, or from public or private research centers.
L'archive ouverte pluridisciplinaire HAL, est destinée au dépôt et à la diffusion de documents scientifiques de niveau recherche, publiés ou non, émanant des établissements d'enseignement et de recherche français ou étrangers, des laboratoires publics ou privés. 


\title{
Probabilistic uncertainty modeling for thermo-mechanical
}

\author{
analysis of plasterboard submitted to fire load \\ Seddik Sakji ${ }^{1}$, Christian Soize ${ }^{2 *} \&$ Jean-Vivien Heck ${ }^{3}$
}

March 10, 2008

\begin{abstract}
The paper deals with a probabilistic modeling of the thermo-mechanical behavior of cardboard-plaster-cardboard (CPC) multilayer plates submitted to fire load. The proposed model takes into account data and model uncertainties. This work is justified by the fact that fire resistance tests of plasterboard-lined partitions are made impossible when their dimensions exceed those of furnaces. A fundamental key to solve such a problem is the development and the experimental validation of a deterministic and probabilistic model of CPC multilayer submitted to fire load. The first step of this work concerns the constitution of an experimental thermo-mechanical data base for a CPC multilayer and for its components. These experimental tests are carried out by the use of a bench test specially designed for this work. The second step is the development of an homogenization thermo-mechanical mean model for the CPC multilayer. The third step is the development of a probabilistic model of uncertainties based on the

\footnotetext{
${ }^{1}$ Assistant Professor, Université Paris-Est, Laboratoire Modélisation et Simulation Multi Echelle, MSME FRE3160 CNRS, 5 bd Descartes, 77454 Marne-la-Vallee, France.

${ }^{2}$ Professor, Université Paris-Est, Laboratoire Modélisation et Simulation Multi Echelle, MSME FRE3160 CNRS, 5 bd Descartes, 77454 Marne-la-Vallee, France. E-mail: christian.soize@univ-paris-est.fr

${ }^{3}$ Research Engineer, Centre scientifique et technique du batiment, 84, Avenue Jean-Jaures, 77447 Champssur-Marne, France.
} 
nonparametric probabilistic approach. Numerical results are compared to the experimental ones.

CE Database subject headings: fire resistance, cardboard-plaster-cardboard multilayer, uncertainties, probabilistic model, experiments, thermal load bench.

\section{Introduction}

Large light partitions (10 meters and more) are non-load bearing elements. They are made of plasterboards screwed on both sides of a metal frame of various constructive configurations. Besides structural requirements such as the resistance to impact loading and collision loads (see European Commitee for Standardization 1994), a light partition must verify various fire resistance criteria such as the carrying out of full scale tests under the ISO 834 thermal loading curve (see International Standards Organization 1975). This last requirement can not be met when the structure dimensions exceed those of the testing furnaces (up to $3 \mathrm{~m}$ ). One way to circumvent the dimensional difficulty consists in evaluating partition behavior by means of experimental and numerical combined approaches. The fundamental key to solve the above problem is to develop and validate with experiments a deterministic and a probabilistic thermo-mechanical model of the plasterboard submitted to fire loads. This paper is only devoted to this aspect and the use of the results presented below in order to analyze large light partitions is introduced in the discussion presented in the last section. A plasterboard (cardboard-plaster-cardboard (CPC) multiplayer) gives a partition higher resistance thanks to the important quantity of capillary and chemically bound water contained in the plaster ( $21 \%$ of its weight, see Axenenko, O. and Thrope, G. 1996). The first step in this paper deals with the identification of the CPC mechanical characteristics under fire and mechanical loads. For that, one adopts a thermomechanical experimental approach taking implicitly into account the hydrous phase. Indeed, 
the mechanical characteristics of the CPC multilayer are determined under the same thermal loading that a partition would receive during conventional resistance tests. For this matter, a new thermal loading bench (TLB) is designed allowing a time evolution thermal load equivalent to the ISO834 function to be reproduced on CPC specimens and then one performs, "quickly", mechanical tests. this means that the test is carried out in two steps. The first step consists in applying a thermal load to a CPC specimen. The duration for step one is between three and ten minutes depending on the the chosen ISO fire load. The second step consists in performing the mechanical test of the specimen of step one for a selected value of the temperature. The duration between the time for which the specimen is extracted from the BST (step 1) and the time corresponding to the end of the mechanical test is thirty seconds as a maximum. Taking into account the brief delay to realize the mechanical test, the evolution of the temperature is negligible and consequently the temperature is not measured. A thermo-mechanical mean model is developed using a homogenization with respect to the thickness of the CPC. In this model, Najar's brittle damage model (see Najar 1987) is introduced for the plaster. A devoted finite element code has been developed and takes into account the evolution of damage within the layers. This mean model is validated with experiments and constitutes a basic model to implement the nonparametric probabilistic approach (Soize 2005) allowing model and data uncertainties to be taken into account. Finally, the numerical results are compared to the experiments.

The outline of the paper is as follows. In the second Section, the procedure of conception is briefly described, the use of the TLB is presented and finally, the thermo-mechanical tests performed on plasterboard and its components are given. The third Section summarizes the CPC thermomechanical mean model of homogenization and the model prediction which are compared to the reference experimental results presented in the second Section. The homogenized thermomechanical probabilistic model and its numerical results are presented in the last Section. 


\section{Experimental identification of the thermo-mechanical properties of the CPC}

To identify the thermo-mechanical properties of the CPC, a thermo-mechanical approach, taking implicitly into account the hydrous phase, is adopted. During ISO 834 equivalent thermal loading, each CPC specimen is taken parallel to the heat source and one side of the specimen is exposed to the former. Consequently, the direction of the heat flux and the hydrous transport are mainly transversal to the specimen. The experimental approach is developed in two steps:

- Thermal loading by means of a thermal load bench (TLB) designed and realized for this work.

- Thermo-mechanical identification by means of a four-point bending test.

\section{Thermal load bench (TLB)}

The TLB (see Fig. 1) reproduces an incidental heat flux equivalent to the one that a partition would receive during a mandatory test using a gas furnace. It is composed of a radiant panel (the heat source) and a mobile cart provided with a specimen holder. The heat flux received by the specimen, hang on the specimen holder, is modified by moving the cart with respect to the radiant panel. Hence, one can reproduce the ISO-thermal-load-equivalent heat flux, not by modifying the flow of combustible gas (as during tests on conventional furnaces), but by modifying the distance between the specimen and the radiant panel. The specimen thermal loading takes place only when the radiant panel reaches its steady state. Therefore the illumination can be considered as constant throughout the test duration. The combination of a heat source used in its steady state and a specimen movement controlled with millimeter precision insures an excellent reproducibility of the thermal load. Furthermore, the ECHAFO software (e.g. see 
Fromy and Curtat 1999) developed in order to model thermal exchanges in fire resistance furnaces, allows us to perform the calculation of the total heat flux received by the specimen surface at different time steps of conventional fire resistance test. First, one characterizes the incidental heat flux received by a fluxmeter at different distances from the radiant panel and then, the cart displacement program is found out in order to reproduce by means of the TLB the incidental heat flux calculated by ECHAFO. Figure 2 compares the proposed TLB density heat flux with the calculated incidental density heat flux on the surface of a plasterboard specimen submitted to the ISO thermal load in a conventional furnace. This figure shows the good re producibility of the TLB thermal loading and validate then its use in the experimental identification of thermo-mechanical characteristics of CPC plates.

\section{Mechanical load bench}

The second step of the experimental protocol concerns the thermo-mechanical identification tests of the CPC multilayer and its components. The values of the Young modulus of the CPC multilayer at different time steps of the ISO thermal load have been identified. Therefore, after having exposed a $0.4 \times 0.4 \mathrm{~m}^{2}$ specimen to a time evolution of a heat flux corresponding to the ISO thermal load using the TLB, a $0.08 \times 0.4 \mathrm{~m}^{2}$ new specimen is cut in the specimen central part. A four-point bending test is then "quickly" performed (see the first Section). The choice of the specimen dimensions (cutting out a $0.08 \times 0.4 \mathrm{~m}^{2}$ new specimen in the thermal loaded specimen) is motivated by a concern to preserve hydrous boundary conditions by avoiding the steam loss in the specimen central part. Concerning cardboard, the same protocol is adopted, the bending test being replaced by a tensile test. These mechanical tests are achieved using an INSTRON press of $5 \cdot 10^{3} \mathrm{~N}$ capacity and a $3 \cdot 10^{-1} \mathrm{~m} / \mathrm{s}$ displacement rate. 


\section{Thermo-mechanical experimental results}

As the plasterboard is an orthotropic material, the bending tests are carried out in the longitudinal (LL), transversal (TT), and diagonal (LT) directions at different steps of the time evolution ISO thermal loading (ambient temperature, 300s ISO, 420s ISO and 600s ISO). For each configuration (mechanical load direction / thermal load time step), six specimens are tested. A measure of the temperature (performed using a thermocouple located in the middle of plasterboard) gives a correspondence between the ISO loading time and the average temperature in the plasterboard. Experimental results show a non linear behavior of the CPC multilayer especially at room temperature. Figure 3 displays the behavior of the CPC plate according to the longitudinal direction for different values of the temperature $\left(20^{\circ} \mathrm{C}, 120^{\circ} \mathrm{C}, 170^{\circ} \mathrm{C}\right.$ and $\left.250^{\circ} \mathrm{C}\right)$. Figure 4 shows the mean value of the experimental Young modulus of the CPC multilayer as a function of temperature. Concerning the thermo-mechanical identification of CPC components, the same experimental protocol have been used. Bending tests performed on plaster samples at different steps of thermal loading show linear-elastic behavior (Fig. 5) with a decreasing stiffness with respect to temperature. As for the cardboard, its behavior shows a light hardening which vanishes when temperature increases (Fig. 6). For the modeling we assume that the two materials are elastic linear.

\section{CPC multilayer thermo-mechanical mean model}

Consider a CPC multilayer composed of three physical layers (subscript $j$ ): cardboard 1 , plaster, cardboard 2. Each physical layer is discretized itself in "numerical" layers (subscript $k$ ) (see Fig.7). The total number of the numerical layers for the composite is denoted by n. Each physical cardboard layer is assumed to be orthotropic while the plaster layer is assumed to be isotropic. 
The elasticity matrix of the constitutive equation of each numerical layer $k(k=1, \ldots, n)$ for the mean model is denoted by $\left[\underline{A}^{k}\right]$ and is a matrix belonging to the set $\mathbb{M}_{5}^{+}(\mathbb{R})$ of all the positive-definite symmetric $(5 \times 5)$ real matrices. The multilayer system is defined in a cartesian coordinate axis (oxyz) where o is located in the composite midplane.

\section{CPC multilayer homogenization with damage}

The usual one dimensional homogenization through the thickness of the multilayer composite yields a matrix equation relating, by means of the homogenized mean matrix $\left[\underline{A}^{g}\right]$, the membrane forces $\mathfrak{n}_{i}, i=\{1,2,6\}$, the bending moments $\mathfrak{m}_{i}, i=\{1,2,6\}$ and the shear forces, $\mathfrak{n}_{i}, i=\{4,5\}$, with the membrane deformations $e_{j}^{m}, j=\{1,2,6\}$, the bending deformations $\kappa_{j}, j=\{1,2,6\}$ and the shear deformations $e_{j}, j=\{4,5\}$ according to Eq. (1).

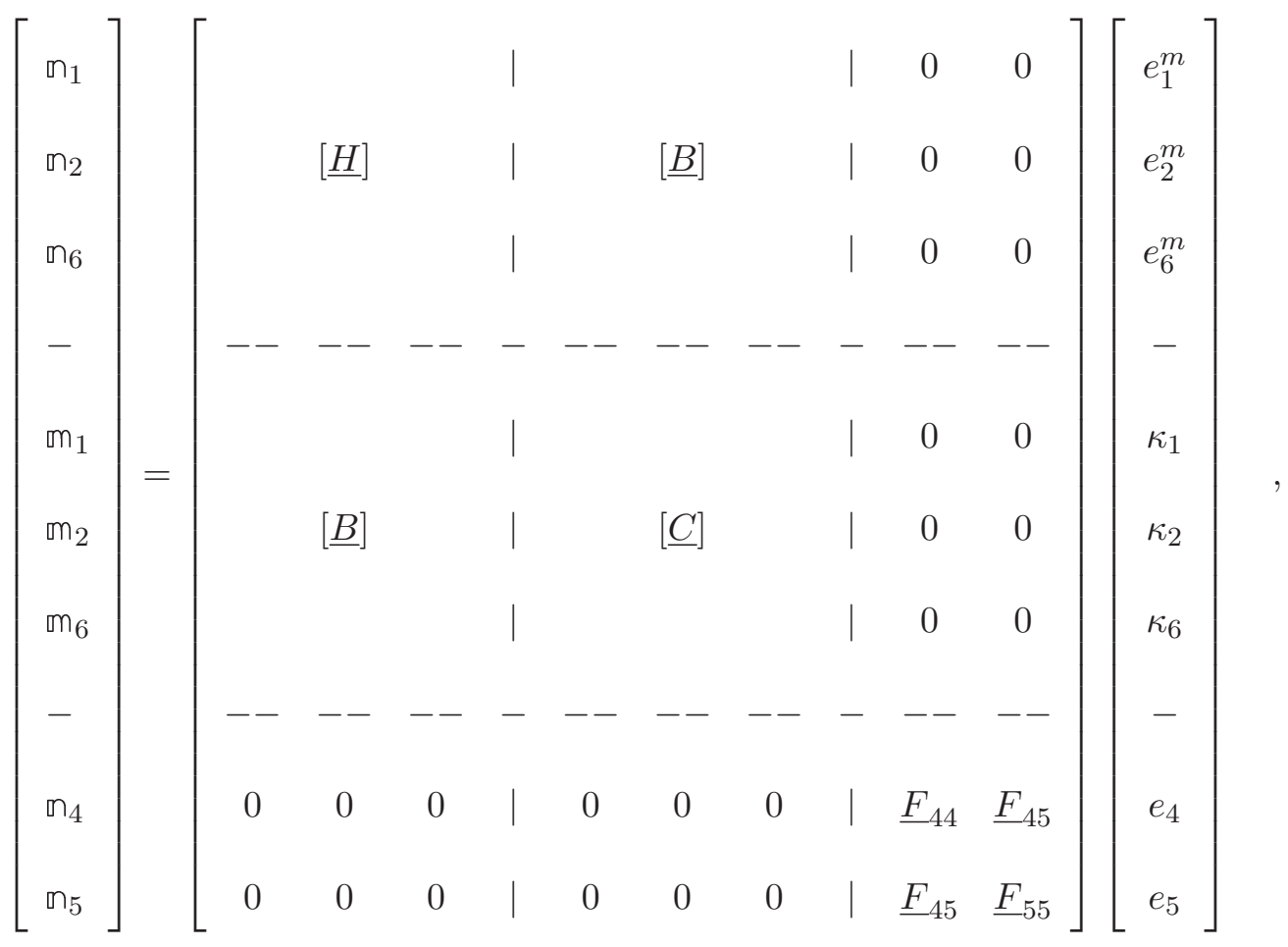


where

$$
\begin{aligned}
& {[\underline{H}]_{i j}=\sum_{k=1}^{n}\left(z_{k}-z_{k-1}\right) \underline{\widetilde{A}}_{i j}^{k}(M, \theta) \quad i \text { and } j=1,2,6} \\
& {[\underline{C}]_{i j}=1 / 3 \sum_{k=1}^{n}\left(z_{k}^{3}-z_{k-1}^{3}\right) \underline{\tilde{A}}_{i j}^{k}(M, \theta) \quad i \text { and } j=1,2,6} \\
& {[\underline{B}]_{i j}=1 / 2 \sum_{k=1}^{n}\left(z_{k}^{2}-z_{k-1}^{2}\right) \underline{\tilde{A}}_{i j}^{k}(M, \theta) \quad i \text { and } j=1,2,6}
\end{aligned},
$$

The Eqs. (2a)-(2d) correspond respectively to the membrane stiffness matrix elements, the bending stiffness matrix elements, the membrane-bending coupling matrix elements and the shear stiffness matrix elements. In these equations, $\theta$ is the temperature and $M=(x, y, z)$ is a point in the $3 \mathrm{D}$ domain. The matrices $\left[\underline{\widetilde{A}}^{k}(M, \theta)\right]$ are such that $\left[\underline{\widetilde{A}}^{k}(M, \theta)\right]=\left(I-\left[\underline{D}^{k}(M, \theta)\right]\right)\left[\underline{A}^{k}(M, \theta)\right]$ in which $\left[\underline{A}^{k}(M, \theta)\right]$ and $\left[\underline{D}^{k}(M, \theta)\right]$ correspond to the stiffness and damage matrices of the layer k, respectively. Finally, one has $\underline{A}_{11}^{k}=E_{x} /\left(1-\nu_{x y} \nu_{y x}\right), \underline{A}_{22}^{k}=E_{y} /\left(1-\nu_{x y} \nu_{y x}\right), \underline{A}_{66}^{k}=G_{x y}$, $\underline{A}_{12}^{k}=\nu_{x y} E_{y} /\left(1-\nu_{x y} \nu_{y x}\right)=\underline{A}_{21}^{k}, \underline{A}_{44}^{k}=G_{y z}, \underline{A}_{55}^{k}=G_{z x}$ and $\underline{A}_{45}^{k}=\underline{A}_{54}^{k}=\underline{A}_{26}^{k}=\underline{A}_{62}^{k}=\underline{A}_{16}^{k}=$ $\underline{A}_{61}^{k}=0$.

\section{Heat transfer analysis}

The mean thermal diffusivity (see Fig. 8) of the CPC plates is identified by an inverse problem performed with a developed finite element code of heat transfer analysis and according to temperature-time tests carried out with the TLB. The CPC thermal conductivity is also identified by thermal experiments at the steady state performed with the TLB. The heat transfer analysis through the thickness of the CPC multilayer (taking into account the identified parameters) allows the temperature in the middle of the different numerical layers of the CPC to be calculated as a function of the thermal loading time (Fig. 9). The mechanical characteristics of 
the CPC layers (Young's modulus) are modeled by an affine function of the mean temperature $\underline{\mathbf{T}}$. The parameters a and $\mathrm{b}$ have been identified with the thermo-mechanical response of the multilayer composite. (This identification yields $a=-3.71$ and $b=2.45$ ). It should be noted that no probabilistic model of uncertainties for the heat transfer analysis is implemented in the model.

\section{Damage model}

As a first approach, a cut-off model was adopted for the plaster layers. For each point of the modeled domain, the damage is equal to zero if the stress is less than the limit stress and equal to 1 otherwise. This first nonlinear approach used for modeling the global behavior of the CPC shows a good qualitative agreement with experimental results at $120^{\circ} \mathrm{C}$ and some differences at room temperature (Sakji et al. 2005). In order to improve the model prediction, a brittle damage model (see Najar 1987) has been implemented for the plaster. According to this model, which is based on strain energy formulation, the damage evolution law of each numerical layer of the plaster can be written as

$$
\beta^{k}=\left\{\begin{array}{ll}
\beta_{0}^{k} \exp \left(\omega^{k}\right) & \text { if } \alpha=0 \\
\beta_{0}^{k}\left(1+\alpha \omega^{k}\right)^{1 / \alpha} & \text { if } \alpha \neq 0
\end{array} .\right.
$$

In Eq. (3) the parameters $\beta_{0}^{k}, \alpha$ and $\omega^{k}$ depend on temperature $\theta$, on the point $\mathrm{M}$ and on $\omega^{k}=W_{p e r f}^{k} / W_{k}^{*}$. The quantity $W_{\text {perf }}^{k}$ is the strain energy of the undamaged material for the layer $k$. The nominal damage energy for the layer $k$, denoted by $W_{k}^{*}$, is defined as the nominal capacity of the material to store energy in the form of internal structural damage. Finally, $\beta_{0}^{k}$ is the initial damage for the layer $k$ and $\alpha$ is a model parameter. As a first assumption, we consider the plaster damage as isotropic and follows the damage evolution law of Eq. (3) in each principal direction. The damage matrix $\left[D^{k}\right]$ related to the numerical layer $k$ of the plaster is 
then written as

$$
\left[D^{k}\right]=\beta^{k}[I], \quad(k=2, \ldots, n-1) \quad .
$$

Finally, the cut-off model is used for the cardboard while a brittle damage model is used for the plasterboard.

\section{Nonlinear thermo-mechanical analysis of the CPC mean model}

A multilayer thin plate theory is used with the constitutive equation Eq. (1). A finite element code is developed for the nonlinear thermo-elastic model. The mechanical response of the CPC is calculated as a function of the external mechanical load corresponding to a four-point bending test. The damage parameters are such that $\alpha_{20}{ }^{\circ} \mathrm{C}=0.1, \beta_{20}{ }^{\circ} \mathrm{C}=0.005,\left(W_{k}^{*}\right)_{20}{ }^{\circ} \mathrm{C}=3.2 \mathrm{KJ} / \mathrm{m}^{3}$ for the temperature $\theta=20^{\circ} \mathrm{C}$ and $\alpha_{120}{ }^{\circ} \mathrm{C}=0.8, \beta_{120^{\circ} \mathrm{C}}=0.005,\left(W_{k}^{*}\right)_{120^{\circ} \mathrm{C}}=2 \mathrm{KJ} / \mathrm{m}^{3}$ for the temperature $\theta=120^{\circ} \mathrm{C}$. Figures 10 and 11 show the comparisons of the experimental results with the numerical simulations for the applied mechanical load as a function of the transversal displacement at $20^{\circ} \mathrm{C}$ and $120^{\circ} \mathrm{C}$ respectively.

\section{CPC multilayer probabilistic model of uncertainties}

We now consider data and model uncertainties in the physical layers (subscript $\mathrm{j}$ ). We have used the nonparametric probabilistic approach (see Soize 2005) in order to take into account these uncertainties. According to this approach, a global random model is constructed using random matrix theory rather than using a probabilistic model of each uncertain parameter (parametric probabilistic approach (e.g. see Ghanem and Spanos 1991)). 


\section{CPC random stiffness matrix}

Each random stiffness matrix $\left[\mathbf{A}^{j}\right]$ is with value in $\mathbf{M}_{5}^{+}(\mathbb{R})$ (plate model). The probability density function of this matrix is constructed following the procedure given in the next section. An homogenization throughout the thickness of the CPC (as for the mean model) is applied to the random multilayer composite and yields the random matrix $\left[\mathbf{A}^{g}\right]$ of the random homogenized constitutive equation

$$
\left[\mathbf{A}^{g}\right]=\operatorname{hom}\left\{\left[\widetilde{\mathbf{A}}^{1}\right], \ldots,\left[\widetilde{\mathbf{A}}^{j}\right]\right\}
$$

\section{Probability density function of the random stiffness matrix of the layer $\mathrm{j}$}

The probability density function of the matrix $\left[\mathbf{A}^{j}\right]$ is constructed using the maximum entropy principle (see Soize 2005). The maximum entropy principle allows the probability density function of a random variable to be constructed by maximizing the entropy function under constraints defined by the available information. The available information related to random matrix $\left[\mathbf{A}^{j}\right]$ is defined as follows:

1. The random matrix is with values in $M_{5}^{+}(\mathbb{R})$.

2. The mean value is such that $\mathcal{E}\left\{\left[\mathbf{A}^{j}\right]\right\}=\left[\underline{A}^{j}\right]$ in which $\mathcal{E}\{\cdot\}$ denotes the mathematical expectation.

3. $\mathcal{E}\left\{\left\|\left[\mathbf{A}^{j}\right]^{-1}\right\|_{F}^{2}\right\}<+\infty$ where $\|[\mathbf{A}]\|_{F}=\left(\operatorname{tr}\left\{[A][A]^{T}\right\}\right)^{1 / 2}$ is the Frobenius norm.

The last constraint insures the existence of a second-order solution of the stochastic problem and then to be consistant with mechanic's considerations.

The random matrix $\left[\mathbf{A}^{j}\right]$ of the $j^{\text {th }}$ layer is written as

$$
\left[\mathbf{A}^{j}\right]=\left[\underline{L}_{A^{j}}\right]^{T}\left[\mathbf{G}_{A^{j}}\right]\left[\underline{L}_{A^{j}}\right]
$$


where $\left[\underline{L}_{A^{j}}\right]$ is a $(5 \times 5)$ real upper triangular matrix such that $\left[\underline{A}^{j}\right]=\left[\underline{L}_{A^{j}}\right]^{T}\left[\underline{L}_{A^{j}}\right]$. The probability distribution function of the random matrix $\left[\mathbf{G}_{A^{j}}\right]$ with values in $\mathbf{M}_{5}^{+}(\mathbb{R})$ is defined by the probability density function $\left[G_{A^{j}}\right] \mapsto p_{\left[\mathbf{G}_{A^{j}}\right]}\left(\left[G_{A^{j}}\right]\right)$ from $\mathbb{M}_{5}^{+}(\mathbb{R})$ in $\mathbb{R}^{+}$with respect to the measure $\widetilde{d} G_{A^{j}}$ such that $\widetilde{d} G_{A^{j}}=2^{n(n-1) / 4} \prod_{1 \leq i \leq k \leq n} d\left[G_{A^{j}}\right]_{i k}$ and such that

$$
p_{\left[\mathbf{G}_{A^{j}}\right]}\left(\left[G_{A^{j}}\right]\right)=\mathbb{1}_{\mathbf{M}_{5}^{+}(\mathbb{R})}\left(\left[G_{A^{j}}\right]\right) \times c_{G_{A^{j}}} \times\left(\operatorname{det}\left[G_{A^{j}}\right]\right)^{3 \frac{1-\delta_{j}^{2}}{\delta_{j}^{2}}} \times \exp \left(-\frac{3}{\delta_{j}^{2}} \operatorname{tr}\left[G_{A^{j}}\right]\right)
$$

in which the normalization positive constant $c_{G_{A^{j}}}$ is written as

$$
c_{G_{A^{j}}}=(2 \pi)^{-5}\left(\frac{3}{\delta_{j}^{2}}\right)^{15 \delta_{j}^{-2}} /\left\{\prod_{l=1}^{n} \Gamma\left(\frac{3}{\delta_{j}^{2}}+\frac{1-l}{2}\right)\right\} .
$$

In Eq. (8), for $z>0, \Gamma(z)$ is the gamma function defined by $\Gamma(z)=\int_{0}^{+\infty} t^{z-1} e^{-t} d t$. The parameter $\delta_{j}$ allows the probability model dispersion of the random matrix $\left[\mathbf{G}_{A^{j}}\right]$ to be controlled (and then the dispersion of the random matrix $\left[\mathbf{A}^{j}\right]$ to be controlled). We have

$$
\delta_{j}=\left\{\mathcal{E}\left\{\left\|\left[\mathbf{G}_{A^{j}}\right]-\left[\underline{G}_{A^{j}}\right]\right\|_{F}^{2}\right\} /\left\|\left[\underline{G}_{A^{j}}\right]\right\|_{F}^{2}\right\}^{1 / 2} .
$$

The parameter $\delta_{j}$ is independent of the random matrix dimension and must be such that

$$
0<\delta_{j}<\sqrt{0.6}
$$

For the Monte-Carlo simulation method, the realizations of the random matrices $[A]^{j}$ are constructed using the following algebraic representation:

$$
\left[\mathbf{G}_{A^{j}}\right]=\left[\mathbf{L}_{j}\right]^{T}\left[\mathbf{L}_{j}\right]
$$

where $\left[\mathbf{L}_{j}\right]$ is an upper random triangular matrix such that:

- Random variables $\left\{\left[\mathbf{L}_{j}\right]_{k k^{\prime}}, \quad k \leq k^{\prime}\right\}$ are independent,

- For $k \leq k^{\prime}$, the random variable $\left[\mathbf{L}_{j}\right]_{k k^{\prime}}$, is such $\left[\mathbf{L}_{j}\right]_{k k^{\prime}}=\sigma_{j} Y_{k k^{\prime}}$, in which $\sigma_{j}=\delta_{j} / \sqrt{6}$ and $Y_{k k^{\prime}}$ is a normalized Gaussian random variable, 
- For $k=k^{\prime},\left[\mathbf{L}_{j}\right]_{k k}=\sigma_{j} \sqrt{2 V_{k j}} \sigma_{j}=\delta_{j} / \sqrt{6}$ where $V_{k j}$ is a gamma random variable which probability density function given by

$$
p_{V_{j}}(\nu)=\mathbb{1}_{\mathbb{R}^{+}}(\nu) \frac{1}{\Gamma\left(3 / \delta_{j}^{2}+(1-k) / 2\right)} \nu^{\frac{3}{\delta_{j}^{2}}+\frac{1-k}{2}} e^{-\nu} .
$$

\section{Finite element discretization of the stochastic boundary value problem}

The nonlinear stochastic boundary value problem is approximated by the usual finite element method (also called stochastic finite element method). The mean global stiffness matrix $([\underline{K}(\mathbf{q}, \underline{\mathbf{T}})])$ of the classic mean finite element modeling $($ i.e. $[\underline{K}(\mathbf{q}, \underline{\mathbf{T}})] \mathbf{q}=\mathbf{f})$ is then replaced by a random matrix $[\mathbf{K}(\mathbf{q}, \underline{\mathbf{T}})]$. The solution $\mathbf{q}$ is no longer deterministic and then becomes a stochastic process denoted by $\mathbb{Q}$. The mean equation is then replaced by the stochastic equation corresponding to the probabilistic model of uncertainties which is written as

$$
[\mathbf{K}(\mathbb{Q}, \underline{\mathbf{T}})] \mathbb{Q}=\mathbf{f}
$$

where $[\mathbf{K}(\mathbb{Q}, \underline{\mathbf{T}})]$ corresponds to the global stiffness matrix constructed by the assembly of the random elementary matrices $\left[\mathbf{K}^{e}(\mathbb{Q}, \underline{\mathbf{T}})\right]$ such that

$$
\left[\mathbf{K}^{e}(\mathbb{Q}, \underline{\mathbf{T}})\right]=\int_{\mathcal{D}_{e}}\left[\mathcal{B}^{e}\left(x_{1}, x_{2}, 0\right)\right]^{T}\left[\mathbf{A}^{g}\left(x_{1}, x_{2}, \mathbb{Q}, \underline{\mathbf{T}}\right)\right]\left[\mathcal{B}^{e}\left(x_{1}, x_{2}, 0\right)\right] d x_{1} d x_{2}
$$

In Eq. (14), $\left[\mathbf{A}^{g}\left(x_{1}, x_{2}, \mathbb{Q}, \underline{\mathbf{T}}\right)\right]$ denotes the homogenized matrix constructed according to Eq. (5) where the updated damage, according to Eq. (3) and Eq. (4), is taken into account using internal iterations. Matrix $\left[\mathcal{B}^{e}\left(x_{1}, x_{2}, 0\right)\right]$ corresponds to the strain interpolation matrix of each finite element defined in the elementary domain $\mathcal{D}_{e}$.

\section{Stochastic solver and convergence}

The Monte-Carlo simulation method is used as the stochastic solver for the random nonlinear equation (i.e. Eq. (13)). For $j=1,2,3$, let $\left[\mathbf{A}^{j}\left(r_{1}\right)\right], \ldots,\left[\mathbf{A}^{j}\left(r_{n_{s}}\right)\right]$ be $n_{s}$ independent realizations of random matrix $\left[\mathbf{A}^{j}\right]$. Each realization $\left\{\left[\mathbf{A}^{j}\left(r_{\ell}\right)\right], j=1,2,3\right\}$ of the physical layers 
(cardboard 1, plaster and cardboard 2) is constructed using the scheme described in the fourth Section and according to Eq. (11), Eq. (7) and Eq. (6). The homogenization process given by Eq. (5) and applied to the random matrices of each numerical layer $k,(k=1, \ldots, n)$ throughout the thickness of the CPC, yields the realization of the random matrix $\left[\mathbf{A}^{g}\left(x_{1}, x_{2}, \mathbb{Q}\left(r_{\ell}, \underline{\mathbf{T}}\right), r_{\ell}\right)\right]$ of the homogenized multilayer. The corresponding realization of Eq. (13) is then written as

$$
\left[\mathbf{K}\left(\mathbb{Q}\left(r_{\ell}\right), \underline{\mathbf{T}}\right)\right] \mathbb{Q}\left(r_{\ell}\right)=\mathbf{f}
$$

Equation (13) is solved by performing increments on load with a load step small enough to take into account damage which is updated according to Eq. (3) of the damage evolution law. The updated damage is taken into account using internal iterations.

The convergence with a respect to the number $n_{s}$ of realizations $r_{1}, \ldots, r_{n_{s}}$ used in the Monte Carlo simulation is studied by analyzing the function

$$
n_{s} \mapsto \operatorname{conv}\left(n_{s}\right)=\left\{\frac{1}{n_{s}} \sum_{\ell=1}^{n_{s}}\left\|\mathbb{Q}\left(r_{\ell}\right)\right\|^{2}\right\}^{1 / 2} .
$$

Let $\mathbb{Q}_{j}$ be the random transversal displacement of the CPC plate at a given node of the mesh. The confidence region with respect to a given probability level $P_{c}$ is defined by the upper and the lower envelopes $\left(q^{+}\right.$and $q^{-}$respectively) such as

$$
\operatorname{Prob}\left(q^{-}<\mathbb{Q}_{j} \leq q^{+}\right)=P_{c}
$$

The estimation of $q^{-}$and $q^{+}$is performed using the sample quantiles. Let $Q_{1}=\mathbb{Q}_{j}\left(r_{1}\right), \ldots, Q_{n_{s}}=$ $\mathbb{Q}_{j}\left(r_{n_{s}}\right), n_{s}$ be independent realizations of the random variable $\mathbb{Q}_{j}$. Let $\widehat{Q}_{1}<\ldots<\widehat{Q}_{n_{s}}$ be the order statistics of the independent variables $Q_{1}, \ldots, Q_{n_{s}}$. Therefore, a possible estimation of the lower and the upper envelopes of $q^{-}$and $q^{+}$is

$$
\begin{aligned}
& q^{-} \simeq \widehat{Q}_{j^{-}} \text {with } j^{-}=f i x\left(n_{s}\left(1-P_{c}\right) / 2\right), \\
& q^{+} \simeq \widehat{Q}_{j^{+}} \text {with } j^{+}=f i x\left(n_{s}\left(1+P_{c}\right) / 2\right),
\end{aligned}
$$


where $f i x(x)$ is the integer part of the real number $x$.

\section{Prediction of the probabilistic model and experimental comparisons}

We consider the boundary value problem corresponding to a four-point bending test and discretized by the stochastic finite element method. We are interested in the random response $\mathbb{Q}$ as a function of the applied mechanical load. Two cases are considered, the first one corresponds to a temperature of $20^{\circ} \mathrm{C}$ (room temperature) and the second one corresponds to $120^{\circ} \mathrm{C}$. The dispersion parameters of the physical layers have been identified with the experimental data using the random response $\mathbb{Q}$ and yields

- $\left(\delta_{\text {plaster }}\right)_{20^{\circ} \mathrm{C}}=0.15$ and $\left(\delta_{\text {cardboard }}\right)_{20^{\circ} \mathrm{C}}=0.08$ at $20^{\circ} \mathrm{C}$,

- $\left(\delta_{\text {plaster }}\right)_{120^{\circ} \mathrm{C}}=0.18$ and $\left(\delta_{\text {cardboard }}\right)_{120^{\circ} \mathrm{C}}=0.09$ at $120^{\circ} \mathrm{C} \quad$.

For each temperature case, the Monte Carlo simulation method is carried out for $n_{s}$ simulations with $\left(n_{s}\right)_{20}{ }^{\circ} \mathrm{C}=350$ and $\left(n_{s}\right)_{120^{\circ} \mathrm{C}}=700$. Figures 12 and 13 are related to the convergence function (Eq. (16)) at $20^{\circ} \mathrm{C}$ and at $120^{\circ} \mathrm{C}$ respectively. Figures 14 and 15 display the comparison of the experiments with the confidence regions calculated for $P_{c}=0.98$ at $20^{\circ} \mathrm{C}$ and $120^{\circ} \mathrm{C}$ respectively.

\section{Discussion an conclusion}

A deterministic nonlinear thermo-mechanical model has been presented for studying a cardboardplaster-cardboard multilayer plate. A new test bench for thermo-mechanical characterization has been specially developed in order to identify the parameters of the thermo-mechanical model and to perform its experimental validation. Due to the high complexity of the thermo-mechanical system induced by the nature of the materials used in the CPC plates (plaster and cardboard), 
there is a significant variability in the real CPC plates induced by the process of fabrication and there are both model uncertainties and data uncertainties in the thermo-mechanical model proposed. Consequently, a probabilistic model of uncertainties has been introduced in order to increase the robustness of the predictions. The dispersion parameters of the probabilistic model of the mechanical properties for the cardboard and for the plaster have experimentally been identified for two temperature steps according to the ISO834 thermal load curve. The confidence region has been constructed to predict the global thermo-mechanical behavior of the CPC multilayer plate submitted to a ISO834 thermal loading. The proposed model with uncertainties can be considered as validated by experiments taking into account the complexity of the thermo-mechanical system.

In this paper, the heat transfer analysis has been performed with deterministic thermal properties, which means that no uncertainties modeling have been taken into account for the thermal properties. It should be noted that another investigation has been developed to analyze the heat transfer with uncertain thermal properties in the context of the probability theory (see Sakji 2006). It has been proven that the temperature field in the CPC plate is sensitive to the uncertainties in the thermal properties but in opposite it has been proven that the statistical fluctuations of the temperature field are not sufficiently large to significantly modify the nonlinear thermo-mechanical behavior of the CPC plate submitted to fire load and mechanical loads. This is the reason why we have presented a simplified version of this work in order to increase its readability. Finally, it should be noted that the uncertainties induced by fire scenario is not considered in the context of this paper because the thermal loading is fixed by the ISO 834 thermal loading curve.

The limitation of the model developed is due to the use of a nonlinear thermo-mechanical model instead of a nonlinear thermo-hydro-chemico-mechanical model. However, it should be 
noted that the hydro-chemical phenomena are implicitly taken into account by the proposed experimental protocol which reproduces the ISO 834 thermal load curve and is also taken into account by the use of the probabilistic model of uncertainties.

Concerning the structural engineering applications, the main conclusions of this paper can be summarized as follows: (1) development of a new experimental protocol for thermo-mechanical characterization of the CPC plate; (2) development of a nonlinear thermo-mechanical model including uncertainties and experimentally validated for CPC plates in spite of the fact that no model existed for such CPC in the literature; (3) the experimental thermo-mechanical measurements on several specimens show small variability of the nonlinear behavior but show a large variability for the ultimate load; this two types of variability are very well predicted by the nonlinear thermo-mechanical model including uncertainties (see fig.(14) and (15)).

The objective of this paper is only focused on the development of experimental and modeling tools to analyze the nonlinear thermo-mechanical response of CPC plates submitted to fire load and mechanical loads. However, these developments constitute a necessary first stage to construct a computational model to analyze the nonlinear thermo-mechanical behavior of a complete large light partition. This aspect requires additional works for which other types of developments must be performed and are not relevant of this paper. Nevertheless, we give below some indications about the methodology which could be used and which is in progress. The first development is related to the connection of the CPC plates to steel frame with studs. A way to solve this problem is to develop a load-displacement constitutive equation for the studs connected to the CPC plate using a local three-dimensional finite element nonlinear thermomechanical model. The second development consists in assembling all the CPC plates on the steel frame with studs and including internal insulation. In a first step, the heat transfer analysis would be done on this partition and the second step would be devoted to the nonlinear thermo- 
mechanical analysis of the partition using load-displacement constitutive equation for the studs and using for each CPC plate the nonlinear thermo-mechanical model including uncertainties and presented in this paper.

\section{Acknowledgement}

This work was supported by the CSTB and the SNIP. The thermal load bench would not be acheived without the help and the effort of P. Fromy, E. Cesmat, D. Pardon, C. Lemerle, P. Rivillion, P. Pimienta and C. Baloche. This help and support are gratefully acknowledged.

\section{Notation}

The following symbols are used in this paper: 
$\left[\underline{A}^{k}\right] \quad$ mean stiffness matrix of the layer $k$;

$\left[\underline{\widetilde{A}}^{k}\right] \quad$ mean damaged stiffness matrix of the layer $k$;

$\left[\underline{A}^{g}\right] \quad$ homogenized stiffness matrix of the multilayer;

$[\underline{B}] \quad$ membrane-bending coupling stiffness matrix;

$[\underline{C}] \quad$ bending stiffness matrix;

$[\underline{F}] \quad$ shear stiffness matrix;

$\left[\mathbf{G}_{A^{j}}\right]$ random matrix germ related to the random matrix $\left[\mathbf{A}^{j}\right] ;$

$[\underline{H}] \quad$ membrane stiffness matrix;

$[I] \quad$ identity matrix;

$J \quad$ number of physical layers;

$\left[\underline{L}_{A^{j}}\right] \quad$ real upper triangular matrix of cholesky decomposed matrix $\left[\underline{A}^{j}\right]$;

$\mathrm{M}^{+}(\mathbb{R})$ set of positive-definite symmetric real matrices;

$n \quad$ number of numerical layers;

$n_{s} \quad$ number of simulations; 
$q^{-}, q^{+} \quad$ lower and upper envelopes of the confidence region;

$r_{\ell} \quad$ the $\ell^{\text {th }}$ realization belonging to the set of all the realizations;

$[S]^{T} \quad$ transpose of matrix $[S]$;

$\operatorname{tr}([S]) \quad$ trace of matrix $[S]$;

$[\underline{S}] \quad$ mean model matrix;

$[\mathbf{S}] \quad$ random matrix;

T vector of the mean temperature in numerical layers;

$\beta^{k} \quad$ damage parameter of the layer $k$;

$\delta^{j} \quad$ dispersion parameter of the random matrix $\left[\mathbf{A}^{j}\right]$;

$\theta \quad$ denotes the temperature ;

$\operatorname{Prob}(X>x)$ probability that $X$ be greater than $x$.

$\mathbb{1}_{\mathbb{R}^{+}}(x) \quad$ equal to 1 if $x \in \mathbb{R}^{+}$and 0 elsewhere;

\section{References}

Axenenko, O. and Thrope, G.(1996). "The modelling of dehydration and stress analysis of gypsum plasterboards exposed to fire." Computational Materials Science, 6:281-294.

European Commitee for Standardization (CEN) (1994). "Eurocode1: actions sur les structures Partie 2-2 : actions générales - Actions sur les structures exposées au feu." European Commitee for Standardization, ENV 1991-2-2(1994).

Fromy, P. and Curtat, M.(1999). "Heat transfer in fire resistance furnaces piloted with thermocouples or plate thermometers." Proc, $6^{\text {th }}$ Int. Symp on Fire Safety Science, IAFSS, M.Curtat, ed., $531-542$.

Ghanem, G. R. and Spanos, P. D.(1991). Stochastic Finite Elements: A Spectral Approach. 
Springer-Verlag, New York.

International Standards Organization (ISO) (1975). "Fire Resistance Tests- Elements of Building Construction." International Standard ISO 834.

Najar. J. (1987). "Continuum damage mechanics, Theory and applications." Continuous Damage of Brittle Solids, D. Krajcinovic and J. Lemaitre, eds. Springer-Verlag, Wien-New York, 223-294.

Sakji, S. (2006). "Probabilistic modeling and experimental validation of heat the transfer analysis and the thermomecanical with damage behavior of a cardboard-plaster-cardboard multilayer submitted to fire loads." in french, Université de Marne-la-Vallee, France.

Sakji, S., Soize, C. and Heck, J.-V. (2005). "Thermo-mechanical model of a cardboard-plastercardboard composite plate submitted to fire load and experiments." Computational Methods and Experiments in Material Characterisation II C.A. BREBBIA, Wessex Institute of Technology UK and A.A. MAMMOLI, University of New Mexico, eds., WIT Transactions on Engineering Sciences, Vol 51, 365p.

Soize, C.(2005). "Random matrix theory for modeling uncertainties in computational mechanics." Computer Methods in Applied Mechanics an Engineering, 194(12-16),1333-1366. 


\section{Captions of figures :}

Fig. 1. Thermal load bench composed of: (1): Specimen holder, (2): mobile cart and (3): radiant panel.

Fig. 2. Incident density heat flux (vertical axis in $\mathrm{KW} / \mathrm{m}^{2}$ ) as a function of time (horizontal axis in second): calculated heat flux (thick solid line), TLB measured heat flux (dashed and marked lines), upper and lower ISO 834 equivalent density flux bounds (dot-dashed and dashed lines respectively).

Fig. 3. Bending test experiments for different values of temperature on $\mathrm{CPC}$ specimens carried out according to the longitudinal direction: graph of the mechanical load (vertical axis in $\mathrm{N}$ ) as a function of the transversal displacement (horizontal axis in $\mathrm{mm}$ ): thick solid lines $\left(250^{\circ} \mathrm{C}\right)$, thin solid lines $\left(170^{\circ} \mathrm{C}\right)$, dot-dashed lines $\left(120^{\circ} \mathrm{C}\right)$, thin dashed lines $\left(20^{\circ} \mathrm{C}\right)$.

Fig. 4. Thermomechanical experiments for the CPC: graph of the mean Young modulus (vertical axis in $\mathrm{MPa}$ ) as a function of the average temperature in the $\mathrm{CPC}$ (horizontal axis in $\left.{ }^{\circ} \mathrm{C}\right)$ : solid line marked "*" $\left(\mathrm{E}_{L L}\right)$, solid line marked "+" $\left(\mathrm{E}_{T T}\right)$, solid line marked " " dashed line $\left(\mathrm{E}_{L T}\right)$.

Fig. 5. Bending tests for different values of temperature carried out on plaster specimens: graph of the mechanical load (vertical axis in $\mathrm{N}$ ) as a function of the transversal displacement (horizontal axis in $\mathrm{mm}$ ): thin solid lines (room temperature), thin dashed line $\left(120^{\circ} \mathrm{C}\right)$, thick lines $\left(170^{\circ} \mathrm{C}\right)$, thick dashed lines $\left(250^{\circ} \mathrm{C}\right)$.

Fig. 6. Tensile tests on cardboard for two different values of temperature: graph of axial stress (in $\mathrm{MPa}$ ) as a function of strain. Solid lines (tests at room temperature), dashed lines (tests after 5min ISO thermal load).

Fig. 7. Schematic representation of cardboard-plaster-cardboard plates. 
Fig. 8. Thermal diffusivity of the CPC (vertical axis in $\left(\mathrm{m}^{2} / \mathrm{s}\right)$ ) as a function of the thermal loading time (horizontal axis in (s)).

Fig. 9. Temperature profiles in the core of CPC plates. Temperature in the middle of numerical layers (Vertical axis in ${ }^{\circ} \mathrm{K}$ ) as a function of the thermal loading time(horizontal axis in $\mathrm{s})$.

Fig. 10. Mechanical applied load (vertical axis in N) as a function of the transversal displacement (horizontal axis in $\mathrm{mm}$ ): experiments (dashed lines), numerical simulation of the mean model at room temperature (thick solid line).

Fig. 11. Mechanical applied load (vertical axis in $\mathrm{N}$ ) as a function of the transversal displacement (horizontal axis in $\mathrm{mm}$ ): experiments (dashed lines), numerical simulation of the mean model at $120^{\circ} \mathrm{C}$ (thick solid line).

Fig. 12. Convergence with respect to the number $n_{s}$ of realizations: graph of the function $n_{s} \mapsto \operatorname{conv}\left(n_{s}\right)$ for numerical simulation at room temperature.

Fig. 13. Convergence with respect to the number $n_{s}$ of realizations: graph of the function $n_{s} \mapsto \operatorname{conv}\left(n_{s}\right)$ for numerical simulation at $120^{\circ} \mathrm{C}$.

Fig. 14. Results for $20^{\circ} \mathrm{C}$ : mechanical applied load (Vertical axis in N) as a function of the transversal displacement (horizontal axis in $\mathrm{mm}$ ): experiments (dashed thin lines), numerical simulations with the mean model (thick solid line), confidence region with the stochastic model (grey region).

Fig. 15. Results for $120^{\circ} \mathrm{C}$ : mechanical applied load (Vertical axis in $\mathrm{N}$ ) as a function of the transversal displacement (horizontal axis in $\mathrm{mm}$ ): experiments (dashed thin lines), numerical simulations with the mean model (thick solid line), confidence region with the stochastic model (grey region). 


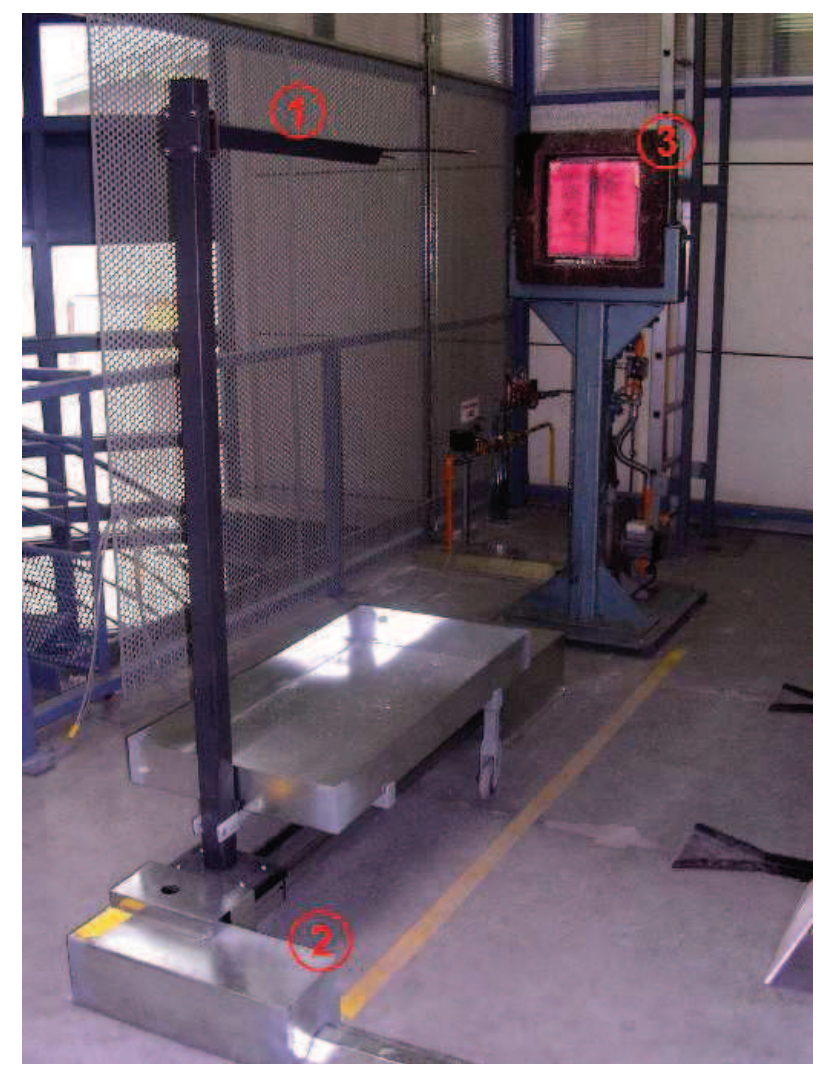

Figure 1: Thermal load bench composed of: (1): Specimen holder, (2): mobile cart and (3): radiant panel. 


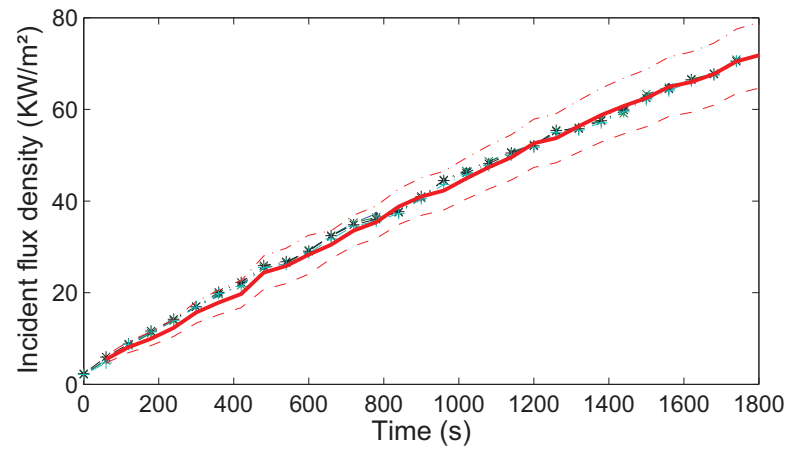

Figure 2: Incident density heat flux (vertical axis in $\mathrm{KW} / \mathrm{m}^{2}$ ) as a function of time (horizontal axis in second): calculated heat flux (thick solid line), TLB measured heat flux (dashed and marked lines), upper and lower ISO 834 equivalent density flux bounds (dot-dashed and dashed lines respectively). 


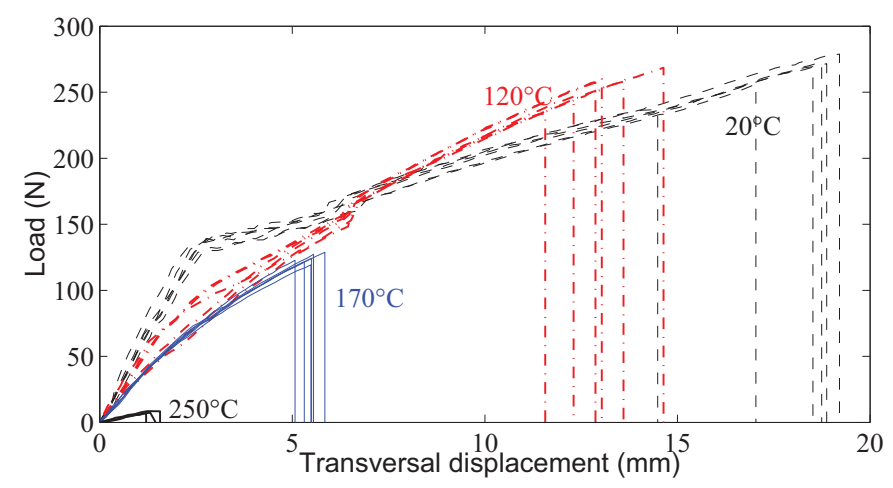

Figure 3: Bending test experiments for different values of temperature on CPC specimens carried out according to the longitudinal direction: graph of the mechanical load (vertical axis in $\mathrm{N}$ ) as a function of the transversal displacement (horizontal axis in $\mathrm{mm})$ : thick solid lines $\left(250^{\circ} \mathrm{C}\right)$, thin solid lines $\left(170^{\circ} \mathrm{C}\right)$, dot-dashed lines $\left(120^{\circ} \mathrm{C}\right)$, thin dashed lines $\left(20^{\circ} \mathrm{C}\right)$. 


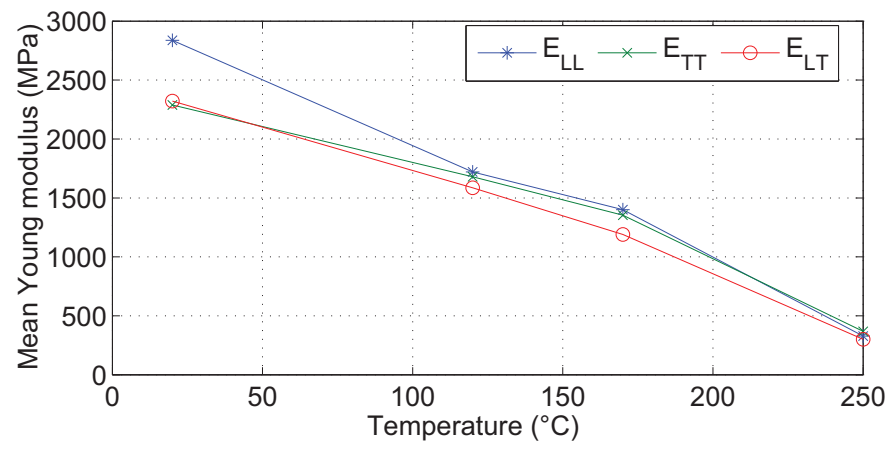

Figure 4: Thermomechanical experiments for the CPC: graph of the mean Young modulus (vertical axis in $\mathrm{MPa}$ ) as a function of the average temperature in the $\mathrm{CPC}$ (horizontal axis in $\left.{ }^{\circ} \mathrm{C}\right)$ : solid line marked "*" $\left(\mathrm{E}_{L L}\right)$, solid line marked "+" $\left(\mathrm{E}_{T T}\right)$, solid line marked "* dashed line $\left(\mathrm{E}_{L T}\right)$. 


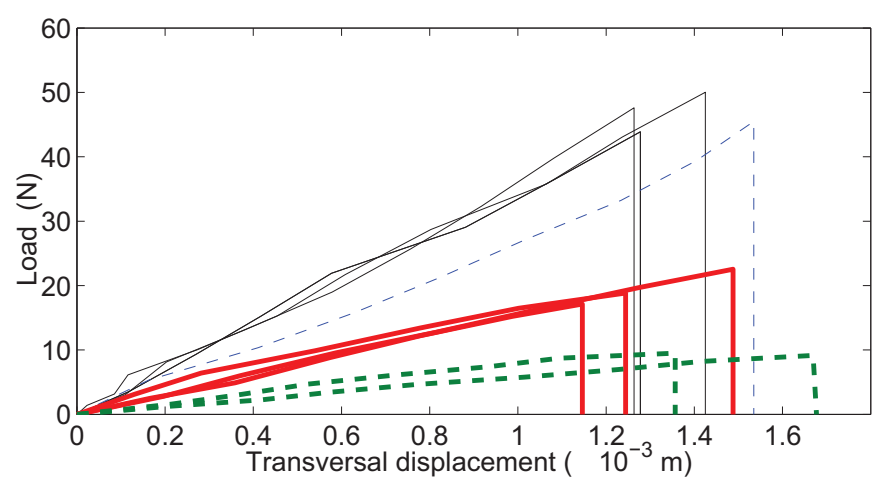

Figure 5: Bending tests for different values of temperature carried out on plaster specimens: graph of the mechanical load (vertical axis in $\mathrm{N}$ ) as a function of the transversal displacement (horizontal axis in $\mathrm{mm}$ ): thin solid lines (room temperature), thin dashed line $\left(120^{\circ} \mathrm{C}\right)$, thick lines $\left(170^{\circ} \mathrm{C}\right)$, thick dashed lines $\left(250^{\circ} \mathrm{C}\right)$. 


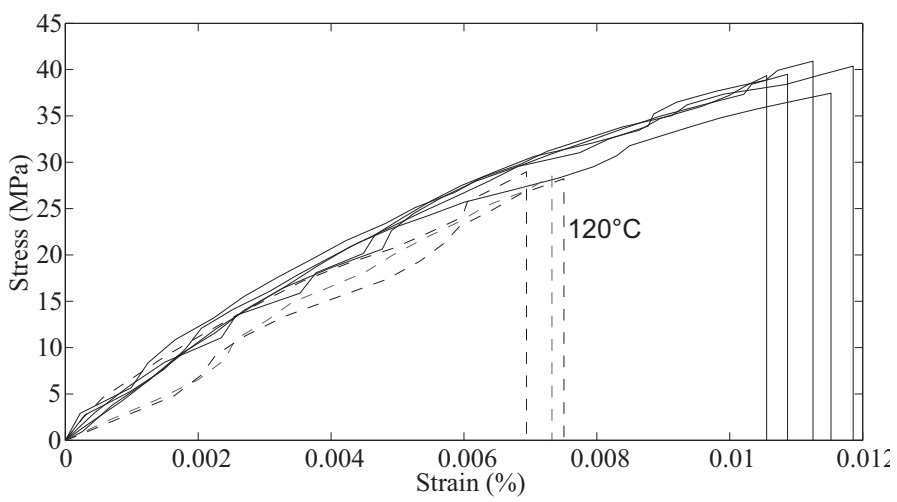

Figure 6: Tensile tests on cardboard for two different values of temperature: graph of axial stress (in MPa) as a function of strain. Solid lines (tests at room temperature), dashed lines (tests after 5min ISO thermal load). 


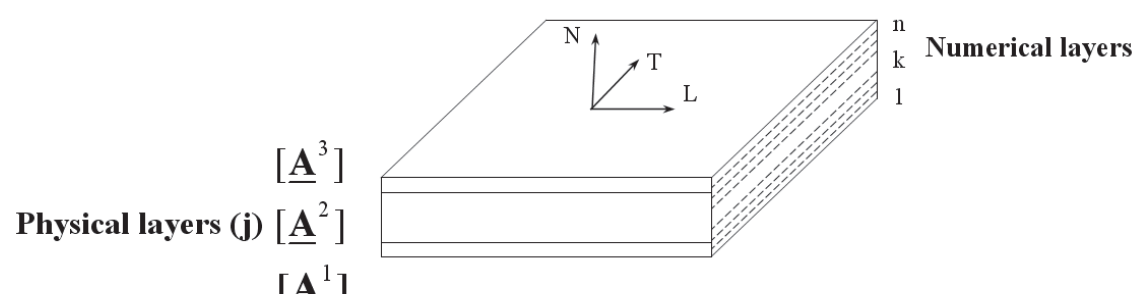

$\left[\underline{\mathbf{A}}^{1}\right]$

Figure 7: Schematic representation of cardboard-plaster-cardboard plates. 


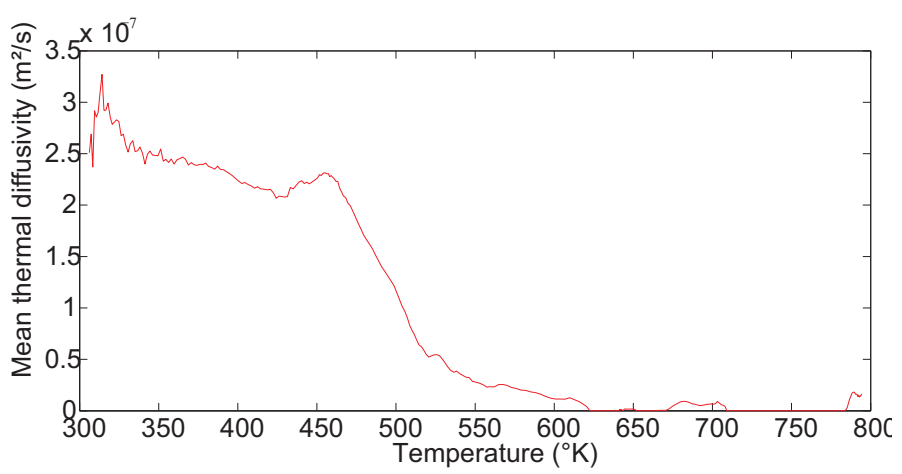

Figure 8: Thermal diffusivity of the CPC (vertical axis in $\left(\mathrm{m}^{2} / \mathrm{s}\right)$ ) as a function of the thermal loading time (horizontal axis in (s)). 


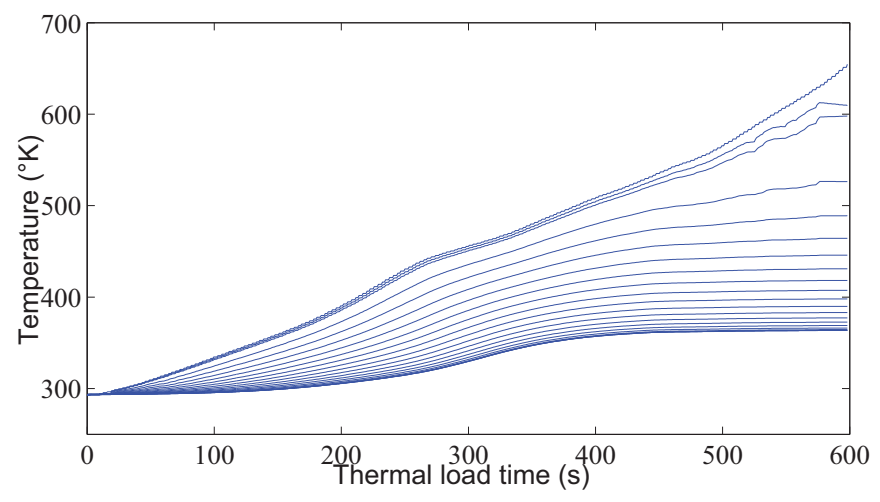

Figure 9: Temperature profiles in the core of CPC plates. Temperature in the middle of numerical layers (Vertical axis in ${ }^{\circ} \mathrm{K}$ ) as a function of the thermal loading time(horizontal axis in s). 


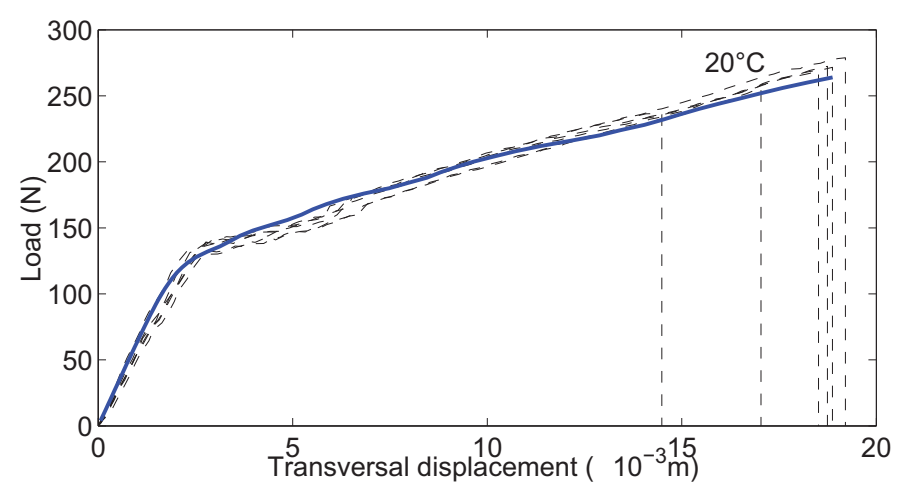

Figure 10: Mechanical applied load (vertical axis in N) as a function of the transversal displacement (horizontal axis in $\mathrm{mm}$ ): experiments (dashed lines), numerical simulation of the mean model at room temperature (thick solid line). 


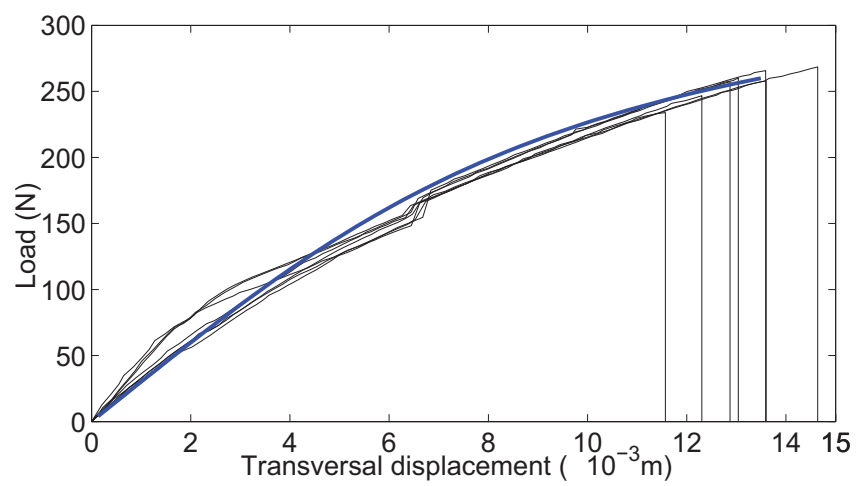

Figure 11: Mechanical applied load (vertical axis in N) as a function of the transversal displacement (horizontal axis in $\mathrm{mm}$ ): experiments (dashed lines), numerical simulation of the mean model at $120^{\circ} \mathrm{C}$ (thick solid line). 


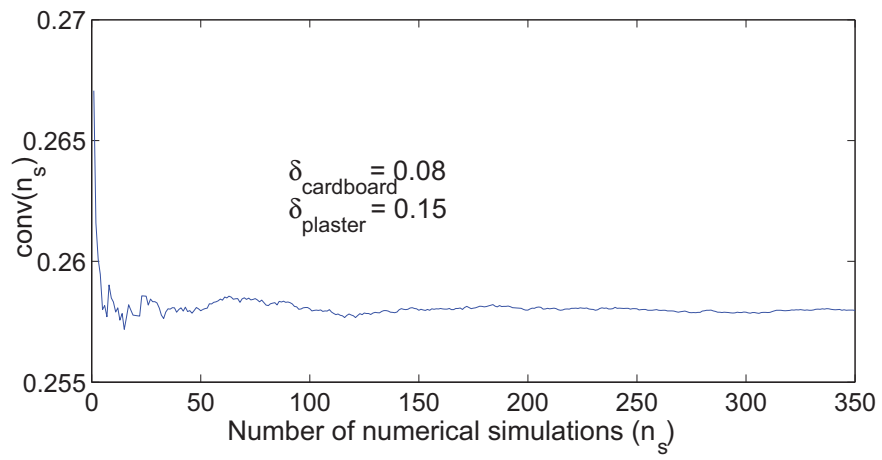

Figure 12: Convergence with respect to the number $n_{s}$ of realizations: graph of the function $n_{s} \mapsto \operatorname{conv}\left(n_{s}\right)$ for numerical simulation at room temperature. 


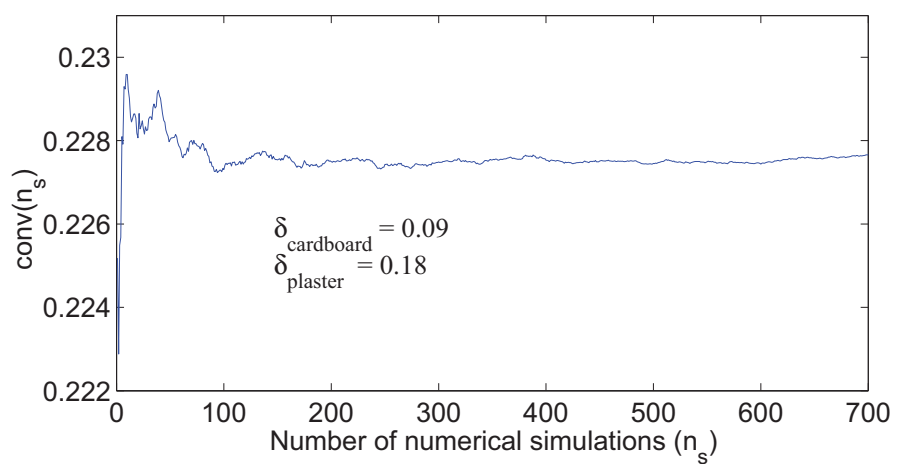

Figure 13: Convergence with respect to the number $n_{s}$ of realizations: graph of the function $n_{s} \mapsto \operatorname{conv}\left(n_{s}\right)$ for numerical simulation at $120^{\circ} \mathrm{C}$. 


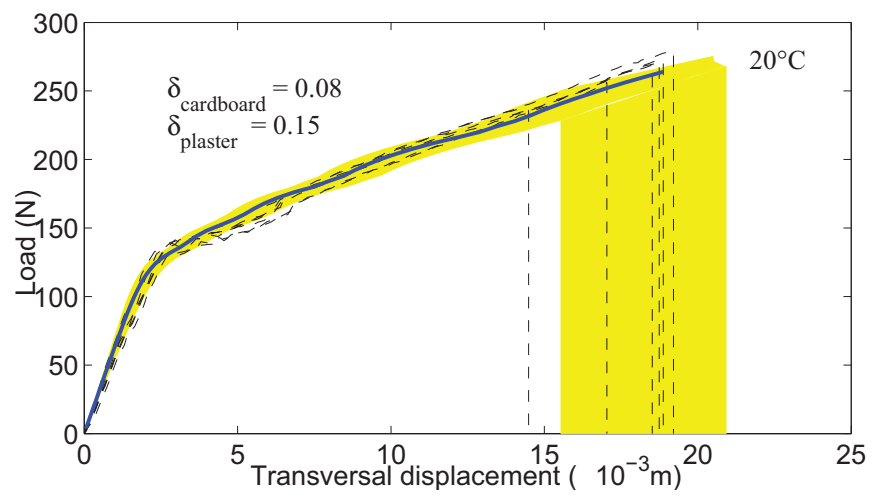

Figure 14: Results for $20^{\circ} \mathrm{C}$ : mechanical applied load (Vertical axis in N) as a function of the transversal displacement (horizontal axis in $\mathrm{mm}$ ): experiments (dashed thin lines), numerical simulations with the mean model (thick solid line), confidence region with the stochastic model (grey region). 


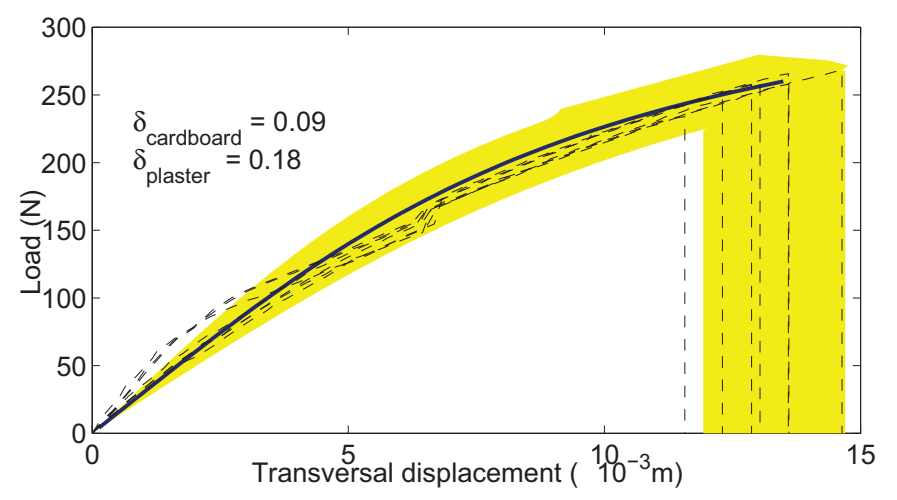

Figure 15: Results for $120^{\circ} \mathrm{C}$ : mechanical applied load (Vertical axis in $\mathrm{N}$ ) as a function of the transversal displacement (horizontal axis in $\mathrm{mm}$ ): experiments (dashed thin lines), numerical simulations with the mean model (thick solid line), confidence region with the stochastic model (grey region). 